\title{
Ramsey numbers of trees versus odd cycles
}

\author{
Matthew Brennan* \\ Department of Electrical Engineering and Computer Science \\ Massachusetts Institute of Technology \\ Massachusetts, U.S.A. \\ brennanm@mit.edu
}

Submitted: Nov 24, 2015; Accepted: Jun 19, 2016; Published: Jul 8, 2016

Mathematics Subject Classifications: 05C55, 05C05, 05C38

\begin{abstract}
Burr, Erdős, Faudree, Rousseau and Schelp initiated the study of Ramsey numbers of trees versus odd cycles, proving that $R\left(T_{n}, C_{m}\right)=2 n-1$ for all odd $m \geqslant 3$ and $n \geqslant 756 m^{10}$, where $T_{n}$ is a tree with $n$ vertices and $C_{m}$ is an odd cycle of length $m$. They proposed to study the minimum positive integer $n_{0}(m)$ such that this result holds for all $n \geqslant n_{0}(m)$, as a function of $m$. In this paper, we show that $n_{0}(m)$ is at most linear. In particular, we prove that $R\left(T_{n}, C_{m}\right)=2 n-1$ for all odd $m \geqslant 3$ and $n \geqslant 25 m$. Combining this with a result of Faudree, Lawrence, Parsons and Schelp yields $n_{0}(m)$ is bounded between two linear functions, thus identifying $n_{0}(m)$ up to a constant factor.
\end{abstract}

\section{Introduction}

The generalized Ramsey number $R(H, K)$ is the smallest positive integer $N$ such for any graph $G$ with at least $N$ vertices either $G$ contains $H$ as a subgraph or its complement $\bar{G}$ contains $K$ as a subgraph, where $H$ and $K$ are any two given graphs. When $H$ and $K$ are complete graphs with $m$ and $n$ vertices respectively, $R(H, K)$ is the classical Ramsey number $R(m, n)$. Classical Ramsey numbers are notoriously difficult to determine. The exact values of many small classical Ramsey numbers including $R(5,5)$ remain unknown. Because of this, Chvátal and Harary proposed to study generalized Ramsey numbers of graphs that are not complete in a series of papers in the early 1970's $[6,7,8]$.

Generalized Ramsey numbers have since been well studied for a variety of graphs, including trees and odd cycles. Let $T_{n}$ be a tree with $n$ vertices and $C_{m}$ denote a cycle of length $m$. Bondy and Erdös showed that $R\left(C_{n}, C_{n}\right)=2 n-1$ for odd $n$ and $R\left(C_{n}, C_{2 r-1}\right)=$ $2 n-1$ if $n>r(2 r-1)$ [1]. Chvátal identified the Ramsey numbers of trees versus complete

*Supported by NSF grant 1358695 and NSA grant H98230-13-1-0273. 
graphs, showing that $R\left(T_{n}, K_{m}\right)=(n-1)(m-1)+1$ for all positive integers $m$ and $n[5]$. Faudree, Lawrence, Parsons and Schelp identified the Ramsey numbers of paths versus odd cycles. If $P_{n}$ denotes a path on $n$ vertices, they showed that $R\left(P_{n}, C_{m}\right)=2 n-1$ for $n \geqslant m \geqslant 3$ and $R\left(P_{n}, C_{m}\right)=\max \{2 n-1, m+\lfloor n / 2\rfloor-1\}$ for $m \geqslant n \geqslant 2$ where $m$ is odd [10]. Faudree, Schelp and Simonovits showed several bounds and exact results on the Ramsey numbers $R\left(T_{n}, C_{\geqslant m}\right)$ where $C_{\geqslant m}$ denotes the family of cycles of length at least $m$ in [11]. These results include that $R\left(T_{n}, C_{\geqslant m}\right) \leqslant 2 m+2 n-7$ for all $m, n \geqslant 3$, $R\left(T_{n}, C_{\geqslant m}\right) \leqslant m+n-2$ if either $m \geqslant n$ or $n \geqslant 432 m^{6}-m^{2}$, and $R\left(T_{n}, C_{\geqslant m}\right)=$ $n+\lfloor m / 2\rfloor-1$ if $T_{n}$ is a tree with maximum degree less than $n-3 m^{2}$ and $n \geqslant 432 m^{6}$. A survey of results about generalized Ramsey numbers can be found in [12].

There have also been lower bounds shown to hold for generalized Ramsey numbers of all graphs. In 1981, Burr showed a lower bound for $R(H, K)$ in terms of the chromatic number $\chi(K)$ of a graph $K$ and its chromatic surplus $s(K)$ - the minimum number of vertices in a color class over all proper vertex colorings of $K$ using $\chi(K)$ colors.

Theorem 1 (Burr [3]). If $s(K)$ is the chromatic surplus of the graph $K$, then for all connected graphs $H$ with $n \geqslant s(K)$ vertices we have

$$
R(H, K) \geqslant(n-1)(\chi(K)-1)+s(K) .
$$

In the case of several of the Ramsey numbers mentioned above, Burr's lower bound is tight. For $K=C_{m}$, Burr's lower bound yields that $R\left(H, C_{m}\right) \geqslant 2 n-1$ where $n=|V(H)|$ since $\chi\left(C_{m}\right)=3$ and $s\left(C_{m}\right)=1$. In 1982, Burr, Erdős, Faudree, Rousseau and Schelp showed that for sufficiently large $n$ and small $\epsilon$, Burr's lower bound on the Ramsey numbers of sparse connected graphs $G$ with at most $(1+\epsilon) n$ edges versus odd cycles $C_{m}$ is tight. Specifically, they proved the following theorem.

Theorem 2 (Burr et al. [4]). If $G$ is a connected graph with $n$ vertices and at most $n\left(1+1 / 42 m^{5}\right)$ edges where $m \geqslant 3$ is odd and $n \geqslant 756 m^{10}$, then $R\left(G, C_{m}\right)=2 n-1$.

This theorem implies the following corollary identifying the Ramsey number of trees versus odd cycles for $n$ very large relative to $m$.

Corollary 3 (Burr et al. [4]). $R\left(T_{n}, C_{m}\right)=2 n-1$ for all odd integers $m \geqslant 3$ and integers $n \geqslant 756 m^{10}$.

Burr, Erdős, Faudree, Rousseau and Schelp asked what the minimum positive integer $n_{0}(m)$ such that this result holds for all $n \geqslant n_{0}(m)$ is as a function of $m$. Their corollary shows that $n_{0}(m)$ is at most a tenth-degree polynomial in $m$. We provide a new approach to examining the Ramsey numbers of trees versus odd cycles and improve this bound, showing that $n_{0}(m)$ is at most linear in $m$. In particular, we prove the following theorem.

Theorem 4. $R\left(T_{n}, C_{m}\right)=2 n-1$ for all odd integers $m \geqslant 3$ and integers $n \geqslant 25 m$.

The result of Faudree, Lawrence, Parsons and Schelp that $R\left(P_{n}, C_{m}\right)=\max \{2 n-$ $1, m+\lfloor n / 2\rfloor-1\}$ for $m \geqslant n \geqslant 2$ where $m$ is odd shows $n_{0}(m) \geqslant 2 m / 3-1$ [10]. Combining this with Theorem 3 yields that $n_{0}(m)$ is bounded between two linear functions. 
In the next two sections, we prove Theorem 4 . We first provide the key lemmas that we use in our proof and then present the proof through a sequence of claims. An important remark is that Burr's lower bound in the case of trees versus odd cycles can be shown by considering the complete bipartite graph $K_{n-1, n-1}$. Because it is bipartite, it does not contain the odd cycle $C_{m}$ as a subgraph. Furthermore, $\overline{K_{n-1, n-1}}$ consists of two connected components of size $n-1$ and therefore does not contain $T_{n}$ as a subgraph. This extremal graph $K_{n-1, n-1}$ will be useful in motivating our proof of Theorem 4 , the last steps of which are devoted to showing that any graph that is any counterexample to Theorem 4 would necessarily have a similar structure to $K_{n-1, n-1}$.

\section{Preliminaries and Lemmas}

We first provide the notation we will adopt on proving Theorem 4. Given a graph $G$, $d_{X}(v)$ denotes the degree of a vertex $v$ in a set $X \subseteq V(G)$ in $G$ and $\overline{d_{X}}(v)$ denotes the degree of $v$ in $X$ in $\bar{G}$, the complement graph of $G$. We similarly let $N_{X}(v)$ and $\overline{N_{X}}(v)$ denote the sets of neighbors of $v$ in the set $X$ in $G$ and $\bar{G}$, respectively. Note that $d_{X}(v)+\overline{d_{X}}(v)=|X|, d_{X}(v)=\left|N_{X}(v)\right|$ and $\overline{d_{X}}(v)=\left|\overline{N_{X}}(v)\right|$ for any set $X \subseteq V(G)$ with $v \notin X$. When the set $X$ is omitted, $X$ is implicitly $V(G)$ where $G$ is the graph in which the vertex $v$ lies. We denote the maximum and minimum degrees of a graph $H$ by $\Delta(H)$ and $\delta(H)$, respectively. Given a subset $S \subseteq V(G)$, we denote the subgraph of $G$ induced by the set $S$ by $G[S]$.

We now present several lemmas that will be used throughout the proof of Theorem 4 . The first two lemmas are extensions of classical results.

Lemma 5. Let $F$ be a forest with $k$ connected components. Let $w_{1}, w_{2}, \ldots, w_{k} \in V(F)$ be vertices from distinct connected components of $F$. Let $H$ be a graph with $\delta(H) \geqslant|V(F)|-1$ and $u_{1}, u_{2}, \ldots, u_{k}$ be distinct vertices in $V(H)$. Then $F$ can be embedded in $H$ such that $w_{i}$ is mapped to $u_{i}$ for all $1 \leqslant i \leqslant k$.

Proof. Begin by mapping $w_{i}$ to $u_{i}$ for all $1 \leqslant i \leqslant k$. Greedily extend this embedding as follows: if $x \in V(F)$ has not been embedded to $H$ but a neighbor $y \in N_{F}(x)$ has been embedded to $z \in V(H)$ then map $x$ to a vertex in $N_{H}(z)$ that has not been embedded to, if such a vertex exists. Since initially a vertex from each connected component of $F$ is mapped to $H$, if all of $F$ has not yet been embedded to $H$ then there must be such a vertex $x$ adjacent to a vertex $y$ that has already been embedded. For the described embedding to fail, there must be a point in this procedure when at most $|V(F)|-1$ vertices have been embedded to and the embedding cannot be extended. Therefore all of $N_{H}(z) \cup\{z\}$ has been embedded to for some $z \in V(H)$. However $\left|N_{H}(z) \cup\{z\}\right| \geqslant \delta(H)+1 \geqslant|V(F)|$, which is a contradiction. Therefore the embedding succeeds, proving the lemma.

The next lemma is also included in our simultaneous work on Ramsey numbers of trees and unicyclic graphs versus fans in [2], where it appears as Lemma 2.

Lemma 6. Given a tree $T$ with $|V(T)| \geqslant 3$, there exists a vertex $v \in V(T)$ satisfying that the vertices of the forest $T-v$ can be partitioned into two disjoint sets $K$ and $H$ such 
that there are no edges between $K$ and $H$ and

$$
\frac{1}{3}(|V(T)|-1) \leqslant|K|,|H| \leqslant \frac{2}{3}(|V(T)|-1) .
$$

Proof. Note that for any vertex $v \in V(T)$, the forest $T-v$ has $d_{T}(v)$ connected components. Now consider the following procedure. Set $v$ initially to be an arbitrary leaf of $T$. At each step, if $T-v$ has a connected component $C$ with $|C| \geqslant|V(T)| / 2$, set $v$ to the unique neighbor $u$ of $v$ in $C$. If no such connected component $C$ exists, terminate the procedure. Observe that $T-u$ has a connected component of size $|V(T)|-|C|$ and one or more connected components with the sum of their sizes equal to $|C|-1$. Therefore either $|C|=|V(T)| / 2$ or the size of the largest connected component of $T-v$ decreases on setting $v$ to $u$. This implies that either the procedure leads to a vertex $v$ such that $T-v$ has a connected component $C$ of size $|C|=|V(T)| / 2$ or terminates at a vertex $v$ such that all connected components of $T-v$ are strictly smaller than $|V(T)| / 2$.

If $v$ satisfies that $T-v$ has a connected component $C$ of size $|C|=|V(T)| / 2$, then let $K=C$ and $H=V(T-v)-C$. We have that $|H|=|V(T)| / 2-1$ and $|K|=|C|=$ $|V(T)| / 2$, which implies the lemma since $|V(T)|$ must be even and hence $|V(T)| \geqslant 4$. Note $d_{T}(v)=1$ is impossible because of the condition on $v$ and since $|V(T)| \geqslant 3$. If $d_{T}(v)=2$, then $T-v$ has two connected components $K$ and $H$ with $|K|+|H|=|V(T)|-1$. Since $|K|,|H|<|V(T)| / 2$, it holds that $|K|=|H|=(|V(T)|-1) / 2$, in which case the lemma is also true.

Now consider the case in which $d_{T}(v)=d \geqslant 3$. Let the connected components of $T-v$ be $C_{1}, C_{2}, \ldots, C_{d}$. Here, it must hold that $|V(T)| \geqslant 4$. Without loss of generality assume that $\left|C_{1}\right| \leqslant\left|C_{2}\right| \leqslant \cdots \leqslant\left|C_{d}\right|<|V(T)| / 2$. Since $d \geqslant 3$ and $\left|C_{1}\right|+\left|C_{2}\right|+\cdots+\left|C_{d}\right|=$ $|V(T)|-1$, we have that $\left|C_{1}\right| \leqslant(|V(T)|-1) / 3$. Let $t$ be the largest integer such that $\left|C_{1}\right|+\left|C_{2}\right|+\cdots+\left|C_{t}\right| \leqslant(|V(T)|-1) / 3$ holds. If $t=d-1$, then $\left|C_{d}\right| \geqslant 2(|V(T)|-1) / 3$ which is impossible because $\left|C_{d}\right|<|V(T)| / 2$. This implies that $t \leqslant d-2$. By definition, $\left|C_{1}\right|+\left|C_{2}\right|+\cdots+\left|C_{t+1}\right|>(|V(T)|-1) / 3$. If $\left|C_{1}\right|+\left|C_{2}\right|+\cdots+\left|C_{t+1}\right| \leqslant 2(|V(T)|-1) / 3$, then letting $K=C_{1} \cup C_{2} \cup \cdots \cup C_{t+1}$ and $H=C_{t+2} \cup C_{t+3} \cup \cdots \cup C_{d}$ yields valid sets $K$ and $H$. If $\left|C_{1}\right|+\left|C_{2}\right|+\cdots+\left|C_{t+1}\right|>2(|V(T)|-1) / 3$, then $(|V(T)|-1) / 3<\left|C_{t+1}\right|<|V(T)| / 2 \leqslant$ $2(|V(T)|-1) / 3$. In this case, letting $K=C_{t+1}$ and $H=C_{1} \cup \cdots \cup C_{t} \cup C_{t+2} \cup \cdots \cup C_{d}$ yields the desired sets $K$ and $H$. This proves the lemma.

Given a graph $G$ and positive integer $n$, let $e x(n, G)$ be the maximum number of edges that a graph on $n$ vertices can have without containing $G$ as a subgraph. This last lemma by Erdos and Galliai determines $e x\left(n, P_{k}\right)$, where $P_{k}$ is a path with $k$ edges and $k+1$ vertices. It can be found in [9] as Theorem 2.6.

Lemma 7 (Erdos and Gallai [9]). For all positive integers $n$ and $k$,

$$
e x\left(n, P_{k}\right) \leqslant \frac{n(k-1)}{2}
$$

where equality holds if and only if $k$ divides $n$, in which case the only graph achieving equality is a union of $\frac{n}{k}$ disjoint copies of $K_{k}$. 


\section{Proof of Theorem 4}

Let $G$ be a graph with $2 n-1$ vertices and assume for contradiction that $G$ does not contain $C_{m}$ as a subgraph and $\bar{G}$ does not contain $T_{n}$ as a subgraph, where $n \geqslant 25 m$. The result $R\left(T_{n}, K_{m}\right)=(n-1)(m-1)+1$ in [5] applied with $m=3$ yields that $R\left(T_{n}, C_{3}\right)=2 n-1$ for all $n$. Therefore, it suffices to prove the result in the case when $m \geqslant 5$.

Our proof of Theorem 4 uses the following key ideas. The lack of a tree in $\bar{G}$ guarantees a large degree vertex in $G$. The absence of an $m$-cycle in $G$ along with this high degree vertex implies there is no path with $m-1$ vertices among its neighbors, which is highly restrictive. The resulting constraints along with two methods for embedding trees yield that there are two large sets $S_{1}$ and $S_{2}$ of vertices in $G$ with a large fraction of the edges between them present. The remainder of the proof is devoted to showing that $G$ must have a similar structure to the extremal graph $K_{n-1, n-1}$. The lack of a length $m$ cycle alternating between these two sets shows that $S_{1} \cup S_{2}$ induces a bipartite subgraph of $G$ and imposes significant constraints on vertices not in $S_{1}$ and $S_{2}$, which are enough to yield a contradiction.

We now proceed to present the proof of Theorem 4 through a series of claims. The first claim bounds the number of edges in a set of neighbors of a vertex. The second claim uses this bound to guarantee that a set of neighbors of a vertex contains a large subset that induces a subgraph with high minimum degree.

Claim 8. For any vertex $v \in V(G)$ and set $X \subseteq V(G)$, the number of edges in the induced subgraph $G\left[N_{X}(v)\right]$ is at most $\frac{1}{2}(m-3) d_{X}(v)$.

Proof. Assume for contradiction that the number of edges in $G\left[N_{X}(v)\right]$ is greater than $\frac{1}{2}(m-3) d_{X}(v)$. By Lemma 7 , there is a path $P$ in $G\left[N_{X}(v)\right]$ with $m-2$ edges and $m-1$ vertices. Since $v$ is adjacent to all vertices of $P$, the vertices $\{v\} \cup P$ form a cycle of length $m$, which is a contradiction. The claim follows.

Claim 9. For any vertex $v \in V(G)$, subset $X \subseteq V(G)$ and positive real number $D$, there is a subset $S \subseteq N_{X}(v)$ such that

$$
|S| \geqslant\left(1-\frac{(m-3)}{2 D}\right) d_{X}(v) \text { and } \delta(\overline{G[S]}) \geqslant|S|-D .
$$

Proof. Consider the following procedure. Begin by setting $R=N_{X}(v)$. At each step, if there is a vertex $u \in R$ such that $d_{R}(u) \geqslant D$, then set $R=R \backslash\{u\}$, otherwise terminate the procedure. At each step, it follows that the number of edges in $G[R]$ decreases by at least $D$. Let $S$ denote the set $R$ obtained once the procedure has terminated. By Claim 8 , the number of edges in $G[R]$ begins no greater than $\frac{1}{2}(m-3) d_{X}(v)$. Therefore the number of steps $t$ of the procedure satisfies that $t \leqslant(m-3) d_{X}(v) / 2 D$. Since $t$ vertices are removed in this procedure, we have that $S$ has size

$$
|S|=d_{X}(v)-t \geqslant\left(1-\frac{(m-3)}{2 D}\right) d_{X}(v) .
$$


Furthermore, for this procedure to terminate it must follow that $\Delta(G[S]) \leqslant D-1$. This implies that

$$
\delta(\overline{G[S]})=|S|-1-\Delta(G[S]) \geqslant|S|-D .
$$

Therefore $S$ has the desired properties, completing the proof of the claim.

If $\delta(\bar{G}) \geqslant n-1$ then by Lemma $5, T_{n}$ can be embedded into $\bar{G}$. Therefore $\delta(\bar{G}) \leqslant n-2$ which implies that $\Delta(G)=2 n-2-\delta(\bar{G}) \geqslant n$. Let $v \in V(G)$ a maximum degree vertex of $G$ with $d(v)=\Delta(G) \geqslant n$. Applying Claim 9 with $D=\sqrt{\frac{1}{2}(m-3) n}$ yields that there is a subset $S_{1} \subseteq N(v)$ such that

$$
\left|S_{1}\right| \geqslant\left(1-\frac{(m-3)}{2 D}\right) d(v) \geqslant n-\sqrt{\frac{1}{2}(m-3) n} .
$$

Furthermore it follows that

$$
\delta\left(\overline{G\left[S_{1}\right]}\right) \geqslant\left|S_{1}\right|-D \geqslant n-\sqrt{2(m-3) n} .
$$

Note that the choice $D=\sqrt{\frac{1}{2}(m-3) n}$ maximizes this lower bound on $\delta\left(\overline{G\left[S_{1}\right]}\right)$. Let $O_{1}=V(G) \backslash S_{1}$. The next claim is the key ingredient to show that there is another large set $S_{2}$ analogous to $S_{1}$ and disjoint from $S_{1}$ in $G$. The proof of this claim applies a method to greedily embed trees used in the proof of Claim 3.3 in [2].

Claim 10. There is a vertex $u \in V(G)$ such that

$$
d_{O_{1}}(u) \geqslant n-\sqrt{\frac{1}{2}(m-3) n}+1 .
$$

Proof. By Lemma 5 , any sub-forest of $T_{n}$ of size at most $\delta\left(\overline{G\left[S_{1}\right]}\right)+1$ can be embedded in $\overline{G\left[S_{1}\right]}$. Since $\bar{G}$ does not contain $T_{n}$ as a subgraph, it must follow that $\delta\left(\overline{G\left[S_{1}\right]}\right) \leqslant n-2$. Note that $T_{n}$ has a connected subtree $H$ on $\delta\left(\overline{G\left[S_{1}\right]}\right)+1$ vertices. For instance, such a subtree is obtained by removing a leaf of $T_{n}$ and repeatedly removing a leaf of the resulting tree until exactly $\delta\left(\overline{G\left[S_{1}\right]}\right)+1$ vertices remain. By Lemma $5, H$ can be embedded to $\overline{G\left[S_{1}\right]}$. Let $R \subseteq S_{1}$ denote the set of vertices that $V(H)$ is mapped to under this embedding.

We now define a procedure to greedily extend this embedding of $H$ to an embedding of $T_{n}$ in $\bar{G}$. At any point in this procedure, let $K$ denote the subgraph of $\bar{G}$ induced by the set of vertices that have so far been embedded to. Initially, $V(K)=R$ and, throughout the procedure, $R \subseteq V(K)$ remains true. Now extend this embedding by repeating the following: if $w_{1}, w_{3} \in V\left(T_{n}\right)$ and $w_{2} \in V(G)$ satisfy that $(1) w_{1} \in V\left(T_{n}\right)$ has been mapped to $w_{2} \in V(K),(2) w_{3} \in V\left(T_{n}\right)$ has not been embedded to $\bar{G}$ and (3) $w_{3}$ is adjacent to $w_{1}$ in $T_{n}$, then map $w_{3}$ to some vertex in $\overline{N_{V(G) \backslash V(K)}}\left(w_{2}\right)$ if it is non-empty. Since $T_{n}$ contains no cycles and $K$ remains connected throughout this procedure, each $w_{3} \in V\left(T_{n}\right)$ that has not yet been embedded to $K$ has at most one neighbor among the vertices $V\left(T_{n}\right)$ that have been embedded to $K$. Furthermore, since $T_{n}$ is connected, if not all of $T_{n}$ has been embedded to $\bar{G}$ then some such $w_{3} \in V\left(T_{n}\right)$ satisfying (1)-(3) must exist. Thus 
this embedding only fails if $\overline{N_{V(G) \backslash V(K)}}\left(w_{2}\right)$ is empty for some $w_{2} \in V(K)$ at some point in the procedure.

Since $\bar{G}$ does not contain $T_{n}$ as a subgraph, this embedding procedure must fail. Therefore $\overline{N_{V(G) \backslash V(K)}}\left(w_{2}\right)=\emptyset$ for some $w_{2} \in V(K)$ where $|V(K)| \leqslant n-1$ at some point during the procedure. Since $R \subseteq V(K)$, it follows that $V(G) \backslash V(K) \subseteq V(G) \backslash R$ and therefore

$$
d_{V(G) \backslash R}\left(w_{2}\right) \geqslant d_{V(G) \backslash V(K)}\left(w_{2}\right) \geqslant|V(G) \backslash V(K)| \geqslant n .
$$

Now note that Claim 9 guarantees that

$$
\left|S_{1} \backslash R\right| \leqslant\left|S_{1}\right|-\delta\left(\overline{G\left[S_{1}\right]}\right)-1 \leqslant D-1=\sqrt{\frac{1}{2}(m-3) n}-1 .
$$

Combining this bound with the previous inequality yields that

$$
d_{O_{1}}\left(w_{2}\right) \geqslant d_{V(G) \backslash R}\left(w_{2}\right)-\left|S_{1} \backslash R\right| \geqslant n-\sqrt{\frac{1}{2}(m-3) n}+1 .
$$

Therefore $w_{2}$ is a vertex with the desired properties, proving the claim.

From this point forward in the proof, let $d=d_{O_{1}}(u)$ where $u$ is the vertex guaranteed by Claim 10 and $d \geqslant n-\sqrt{\frac{1}{2}(m-3) n}+1$. Now applying Claim 9 with $D=\sqrt{\frac{1}{2}(m-3) d}$ yields that there is a subset $S_{2} \subseteq N_{O_{1}}(u)$ such that

$$
\left|S_{2}\right| \geqslant\left(1-\frac{(m-3)}{2 D}\right) d=d-\sqrt{\frac{1}{2}(m-3) d} .
$$

Furthermore it follows that

$$
\delta\left(\overline{G\left[S_{2}\right]}\right) \geqslant\left|S_{2}\right|-D \geqslant d-\sqrt{2(m-3) d} .
$$

Note that since $S_{2} \subseteq N_{O_{1}}(u) \subseteq O_{1}$, it necessarily follows that $S_{1}$ and $S_{2}$ are disjoint. We remark that $S_{1}$ and $S_{2}$ are symmetric other than the fact that the lower bounds on $\left|S_{2}\right|$ and $\delta\left(\overline{G\left[S_{2}\right]}\right)$ are weaker than those on $\left|S_{1}\right|$ and $\delta\left(\overline{G\left[S_{1}\right]}\right)$. In order to obtain the bound $n \geqslant 25 m$, we do not treat $S_{1}$ and $S_{2}$ completely symmetrically, which would entail discarding the better lower bounds for $S_{1}$. The next claim shows that a large fraction of the edges are present between $S_{1}$ and $S_{2}$.

Claim 11. Each $w \in S_{1}$ satisfies that $\overline{d_{S_{2}}}(w)<n-\delta\left(\overline{G\left[S_{1}\right]}\right)-1$ and each $w \in S_{2}$ satisfies that $\overline{d_{S_{1}}}(w)<n-\delta\left(\overline{G\left[S_{2}\right]}\right)-1$.

Proof. Before proving this claim, we first show the two inequalities

$$
(n-1) / 2 \leqslant \delta\left(\overline{G\left[S_{2}\right]}\right) \quad \text { and } \quad 2(n-1) / 3 \leqslant \delta\left(\overline{G\left[S_{1}\right]}\right) .
$$


Here we apply the lower bound on $n$ in terms of $m$. In particular, if $n \geqslant 18 m$, we have the following inequalities

$$
\begin{aligned}
\delta\left(\overline{G\left[S_{1}\right]}\right) & \geqslant n-\sqrt{2(m-3) n}>2 n / 3>2(n-1) / 3, \quad \text { and } \\
d & \geqslant n-\sqrt{\frac{1}{2}(m-3) n}+1>5 n / 6 .
\end{aligned}
$$

Applying $d>5 n / 6 \geqslant 15 m$ to the lower bound on $\delta\left(\overline{G\left[S_{2}\right]}\right)$ yields that

$$
\begin{aligned}
\delta\left(\overline{G\left[S_{2}\right]}\right) & \geqslant d-\sqrt{2(m-3) d}>\left(1-\frac{2}{\sqrt{30}}\right) d \\
& >\frac{5}{6}\left(1-\frac{2}{\sqrt{30}}\right) n>(n-1) / 2
\end{aligned}
$$

since $\frac{5}{6}\left(1-\frac{2}{\sqrt{30}}\right) \approx 0.53$. We now proceed to the proof of the claim.

We first show that each $w \in S_{2}$ satisfies that $\overline{d_{S_{1}}}(w)<n-\delta\left(\overline{G\left[S_{2}\right]}\right)-1$. Assume for contradiction that some $w \in S_{2}$ satisfies that $\overline{d_{S_{1}}}(w) \geqslant n-\delta\left(\overline{G\left[S_{2}\right]}\right)-1$. By Lemma 6 , there is some vertex $x \in V\left(T_{n}\right)$ such that there is a partition $K \cup H$ of the vertices of the forest $T_{n}-x$ such that there are no edges between $K$ and $H$ in $T_{n}$ and $(n-1) / 3 \leqslant$ $|K|,|H| \leqslant 2(n-1) / 3$. Without loss of generality assume that $|H| \leqslant(n-1) / 2 \leqslant|K|$.

First suppose that $d_{K}(x) \leqslant \overline{d_{S_{1}}}(w)$. Consider the following embedding of $T_{n}$ into $\bar{G}$. Note that since $K$ and $H$ are unions of connected components of $T_{n}-x$, it follows that $H \cup\{x\}$ is a subtree of $T_{n}$. Since

$$
|H \cup\{x\}| \leqslant 1+(n-1) / 2 \leqslant \delta\left(\overline{G\left[S_{2}\right]}\right)+1,
$$

by Lemma 5 we have that $H \cup\{x\}$ can be embedded in $\overline{G\left[S_{2}\right]}$ such that $x$ is mapped to $w$. Furthermore, note that $K$ is the union of connected components of $T_{n}-x, K$ is a sub-forest of $T_{n}$ and $N_{K}(x)$ consists of exactly one vertex from each of the connected components of $K$. Now note that since

$$
|K| \leqslant 2(n-1) / 3 \leqslant \delta\left(\overline{G\left[S_{1}\right]}\right)+1
$$

by Lemma 5 we have that $K$ can be embedded to $\overline{G\left[S_{1}\right]}$ such that $N_{K}(x)$ is mapped to $k=d_{K}(x)$ distinct vertices $y_{1}, y_{2}, \ldots, y_{k}$ in $\overline{N_{S_{1}}}(w)$. Note this is possible since $d_{K}(x) \leqslant$ $\overline{d_{S_{1}}}(w)$. This yields a successful embedding of $T_{n}$ in $\bar{G}$, which is a contradiction since $\bar{G}$ does not contain $T_{n}$ as a subgraph.

Now suppose that $d_{K}(x)>\overline{d_{S_{1}}}(w) \geqslant n-\delta\left(\overline{G\left[S_{2}\right]}\right)-1$. Observe that $K$ is the union of $d_{K}(x)$ connected components of $T_{n}-x$. Let $C$ be the union of $d_{K}(x)-\overline{d_{S_{1}}}(w)$ of these connected components. Let $K^{\prime}=K \backslash C$ and $H^{\prime}=H \cup C$. Note that $d_{K^{\prime}}(x)=\overline{d_{S_{1}}}(w) \geqslant$ $n-\delta\left(\overline{G\left[S_{2}\right]}\right)-1$ and that

$$
n-\delta\left(\overline{G\left[S_{2}\right]}\right)-1 \leqslant d_{K^{\prime}}(x) \leqslant\left|K^{\prime}\right| \leqslant 2(n-1) / 3<\delta\left(\overline{G\left[S_{1}\right]}\right)+1 .
$$


The lower bound on $\left|K^{\prime}\right|$ implies that

$$
\left|H^{\prime} \cup\{x\}\right|=n-\left|K^{\prime}\right| \leqslant \delta\left(\overline{G\left[S_{2}\right]}\right)+1 .
$$

Now applying the embedding described above to $K^{\prime}$ and $H^{\prime}$ in place of $K$ and $H$ yields the same contradiction.

The same method shows that each $w \in S_{1}$ satisfies that $\overline{d_{S_{2}}}(w)<n-\delta\left(\overline{G\left[S_{1}\right]}\right)-1$. Specifically, if $\overline{d_{S_{2}}}(w) \geqslant n-\delta\left(\overline{G\left[S_{1}\right]}\right)-1$ for some $w \in S_{1}$, embedding the tree $K \cup\{x\}$ to $\overline{G\left[S_{1}\right]}$ with $x$ mapped to $w$ and embedding the forest $H$ to $\overline{G\left[S_{2}\right]}$ as in the argument above yields a contradiction. This proves the claim.

From this point forward in the proof of Theorem 4 , let $m=2 \ell+1$. The next two claims together complete the proof that the sets $S_{1}$ and $S_{2}$ induce a nearly complete bipartite subgraph of $G$, further showing that the structure of $G$ is close to that of the extremal graph $K_{n-1, n-1}$.

Claim 12. If the vertices $x, y \in S_{1}$, then $\left|N_{S_{2}}(x) \cap N_{S_{2}}(y)\right|>\ell$ and, if $x, y \in S_{2}$, then $\left|N_{S_{1}}(x) \cap N_{S_{1}}(y)\right|>\ell$.

Proof. If $x, y \in S_{1}$, then since $\overline{d_{S_{2}}}(x), \overline{d_{S_{2}}}(y)<n-\delta\left(\overline{G\left[S_{1}\right]}\right)-1$ we have that

$$
\begin{aligned}
\left|N_{S_{2}}(x) \cap N_{S_{2}}(y)\right| & \geqslant\left|S_{2}\right|-\overline{d_{S_{2}}}(x)-\overline{d_{S_{2}}}(y) \\
& >\left|S_{2}\right|-2\left(n-\delta\left(\overline{G\left[S_{1}\right]}\right)-1\right) .
\end{aligned}
$$

Similarly, if $x, y \in S_{2}$, then since $\overline{d_{S_{1}}}(x), \overline{d_{S_{1}}}(y)<n-\delta\left(\overline{G\left[S_{2}\right]}\right)-1$ we have that

$$
\begin{aligned}
\left|N_{S_{1}}(x) \cap N_{S_{1}}(y)\right| & \geqslant\left|S_{1}\right|-\overline{d_{S_{1}}}(x)-\overline{d_{S_{1}}}(y) \\
& >\left|S_{1}\right|-2\left(n-\delta\left(\overline{G\left[S_{2}\right]}\right)-1\right) .
\end{aligned}
$$

We will show that both of these lower bounds are at least $\ell=(m-1) / 2$ when $n \geqslant 25 m$. Assume that $n \geqslant \frac{1}{2} c^{2} m$ and note the following inequalities

$$
\begin{aligned}
\left|S_{1}\right| & \geqslant n-\sqrt{\frac{1}{2}(m-3) n}>\left(1-\frac{1}{c}\right) n, \\
\delta\left(\overline{G\left[S_{1}\right]}\right) & \geqslant n-\sqrt{2(m-3) n}>\left(1-\frac{2}{c}\right) n \\
d & \geqslant n-\sqrt{\frac{1}{2}(m-3) n}+1>\left(1-\frac{1}{c}\right) n \geqslant \frac{1}{2} c(c-1) m, \\
\left|S_{2}\right| & \geqslant d-\sqrt{\frac{1}{2}(m-3) d}>\left(1-\frac{1}{\sqrt{c(c-1)}}\right) d \\
& >\left(1-\frac{1}{c}-\frac{\sqrt{c-1}}{c \sqrt{c}}\right) n, \quad \text { and } \\
\delta\left(\overline{G\left[S_{2}\right]}\right) & \geqslant d-\sqrt{2(m-3) d} \geqslant\left(1-\frac{1}{c}-\frac{2 \sqrt{c-1}}{c \sqrt{c}}\right) n .
\end{aligned}
$$


These inequalities imply that

$$
\begin{aligned}
& \left|S_{2}\right|-2\left(n-\delta\left(\overline{G\left[S_{1}\right]}\right)-1\right)>\left(1-\frac{5}{c}-\frac{\sqrt{c-1}}{c \sqrt{c}}\right) n \geqslant \frac{n}{2 c^{2}} \geqslant \frac{m}{2}>\ell \text { and } \\
& \left|S_{1}\right|-2\left(n-\delta\left(\overline{G\left[S_{2}\right]}\right)-1\right)>\left(1-\frac{3}{c}-\frac{4 \sqrt{c-1}}{c \sqrt{c}}\right) n \geqslant \frac{n}{2 c^{2}} \geqslant \frac{m}{2}>\ell
\end{aligned}
$$

as long as we have the inequalities

$$
\begin{aligned}
& 1-\frac{5}{c}-\frac{\sqrt{c-1}}{c \sqrt{c}} \geqslant \frac{1}{2 c^{2}} \text { and } \\
& 1-\frac{3}{c}-\frac{4 \sqrt{c-1}}{c \sqrt{c}} \geqslant \frac{1}{2 c^{2}} .
\end{aligned}
$$

Since $\sqrt{c-1}<\sqrt{c}$, rearranging yields that these inequalities hold if $2 c(c-7) \geqslant 1$, which is true when $c^{2}=50$. The claim follows.

Claim 13. There are no edges in $G\left[S_{1}\right]$ and $G\left[S_{2}\right]$.

Proof. Now assume for contradiction that there is an edge $x y$ in $G\left[S_{1}\right]$. Let $z_{1}, z_{2}, \ldots, z_{\ell+1}$ be any distinct vertices of $S_{1}$ such that $z_{1}=x$ and $z_{\ell+1}=y$. By Claim 12, there are at least $\ell$ vertices of $S_{2}$ adjacent to both $z_{i}$ and $z_{i+1}$ for each $1 \leqslant i \leqslant \ell$. Therefore we may choose distinct vertices $w_{1}, w_{2}, \ldots, w_{\ell}$ in $S_{2}$ such that $w_{i}$ is adjacent to both $z_{i}$ and $z_{i+1}$ for all $1 \leqslant i \leqslant \ell$. Now note that the vertices $z_{1}, w_{1}, z_{2}, w_{2}, \ldots, z_{\ell}, w_{\ell}, z_{\ell+1}$ form a cycle of length $2 \ell+1=m$ in $G$, which is a contradiction. A symmetric argument shows that there are no edges in $G\left[S_{2}\right]$. This completes the proof of the claim.

Now let $U=V(G) \backslash\left(S_{1} \cup S_{2}\right)$. The next claim is the final ingredient necessary to construct a successful embedding of $T_{n}$ to $\bar{G}$.

Claim 14. Each $w \in U$ is adjacent to vertices in at most one of $S_{1}$ and $S_{2}$.

Proof. Assume for contradiction that some vertex $w \in U$ is adjacent to $x \in S_{1}$ and $y \in S_{2}$. Since $d_{S_{1}}(y)=\left|N_{S_{1}}(y)\right|>\ell \geqslant 2$ by Claim 12, $y$ has a neighbor $z \in S_{1}$ where $z \neq x$. Now let $z_{1}, z_{2}, \ldots, z_{\ell}$ be any distinct vertices of $S_{1}$ such that $z_{1}=z$ and $z_{\ell}=x$. By Claim 12 , it follows that there are at least $\ell$ vertices of $S_{2}$ adjacent to both $z_{i}$ and $z_{i+1}$ for each $1 \leqslant i \leqslant \ell-1$. Therefore we may choose distinct vertices $w_{1}, w_{2}, \ldots, w_{\ell-1}$ in $S_{2}$ such that $w_{i}$ is adjacent to both $z_{i}$ and $z_{i+1}$ and $w_{i} \neq y$ for all $1 \leqslant i \leqslant \ell-1$. Now note that the vertices $w, y, z_{1}, w_{1}, z_{2}, w_{2}, \ldots, z_{\ell-1}, w_{\ell-1}, z_{\ell}$ forms a cycle of length $2+2(\ell-1)+1=m$ in $G$, which is a contradiction. This proves the claim.

By Claim 14, there are sets $U_{1}$ and $U_{2}$ such that $U=U_{1} \cup U_{2}$ and no vertex in $U_{i}$ is adjacent to a vertex of $S_{i}$ for $i=1,2$. It follows that $\left|S_{1} \cup U_{1}\right|+\left|S_{2} \cup U_{2}\right| \geqslant|V(G)|=2 n-1$ and therefore either $\left|S_{1} \cup U_{1}\right| \geqslant n$ or $\left|S_{2} \cup U_{2}\right| \geqslant n$.

First suppose that $\left|S_{1} \cup U_{1}\right| \geqslant n$. Since $T_{n}$ is a tree, it is bipartite and admits a bipartition $V\left(T_{n}\right)=A \cup B$ where $|A| \geqslant|B|$ and thus $|A| \geqslant n / 2$. Now consider the 
following embedding of $T_{n}$ to $\overline{G\left[S_{1} \cup U_{1}\right]}$. If $\left|S_{1}\right| \geqslant n$ then map the vertices of $T_{n}$ arbitrarily to distinct vertices in $S_{1}$. Otherwise, map $n-\left|S_{1}\right|$ vertices in $A$ to distinct vertices in $U_{1}$ and the remaining $\left|S_{1}\right|$ vertices of $T_{n}$ to distinct vertices in $S_{1}$. Note that this is possible since $n-\left|S_{1}\right| \leqslant \sqrt{\frac{1}{2}(m-3) n} \leqslant n / 2 \leqslant|A|$ since $n \geqslant 2 m-6$. Since each vertex in $S_{1}$ is not adjacent to all other vertices in $S_{1} \cup U_{1}$ and $A$ is an independent set of $T_{n}$, this is a valid embedding. This contradicts the fact that $\bar{G}$ does not contain $T_{n}$ as a subgraph. We arrive at a symmetric contradiction when $\left|S_{2} \cup U_{2}\right| \geqslant n$. This proves Theorem 4 .

\section{Conclusions and Future Work}

The primary direction for further work is to determine exactly the number $n_{0}(m)$. Our work and the path-odd cycle result of Faudree, Lawrence, Parsons and Schelp in [10] show that $2 m / 3-1 \leqslant n_{0}(m) \leqslant 25 m[10]$. Another possible direction for future work would be to extend the methods shown here to families of sparse graphs other than trees, such as unicyclic graphs.

\section{Acknowledgements}

This research was conducted at the University of Minnesota Duluth REU and was supported by NSF grant 1358695 and NSA grant H98230-13-1-0273. The author thanks Joe Gallian for suggesting the problem and Levent Alpoge and Joe Gallian for helpful comments on the manuscript. The author also thanks Péter Csikvári for suggesting Lemma 7 to improve the bound in Claim 8 .

\section{References}

[1] J.A. Bondy and P. Erdős, Ramsey numbers for cycles in graphs, J. Combin. Theory, Ser. B 14 (1973), no. 1, 46-54.

[2] M. Brennan, Ramsey numbers of trees and unicyclic graphs versus fans, preprint (2015), arXiv:1511.07306.

[3] S.A. Burr, Ramsey numbers involving graphs with long suspended paths, J. London Math. Soc. 2 (1981), no. 3, 405-413.

[4] S.A. Burr, P. Erdős, R.J. Faudree, C.C. Rousseau, and R.H. Schelp, Ramsey numbers for the pair sparse graph-path or cycle, Trans. Amer. Math. Soc. 269 (1982), no. 2, 501-512.

[5] V. Chvátal, Tree-complete graph Ramsey numbers, J. Graph Theory 1 (1977), no. 1, 93-93.

[6] V. Chvátal and F. Harary, Generalized Ramsey theory for graphs. II. small diagonal numbers, Proc. Amer. Math. Soc. 32 (1972), no. 2, 389-394.

[7] V. Chvátal and F. Harary, Generalized Ramsey theory for graphs. III. small offdiagonal numbers, Pacific J. Math. 41 (1972), no. 2, 335-345. 
[8] V. Chvátal and F. Harary, Generalized Ramsey theory for graphs. I. diagonal numbers, Periodica Math. Hungarica 3 (1973), no. 1-2, 115-124.

[9] P. Erdős and T. Gallai, On maximal paths and circuits of graphs, Acta Math. Acad. Sci. Hungarica 10 (1959), no. 3-4, 337-356.

[10] R.J. Faudree, S.L. Lawrence, T.D. Parsons, and R.H. Schelp, Path-cycle Ramsey numbers, Discrete Math. 10 (1974), no. 2, 269-277.

[11] R.J. Faudree, R.H. Schelp and M. Simonovits, On some Ramsey type problems connected with paths, cycles and trees, Ars Combinatoria, 29A (1990) 97-106.

[12] S.P. Radziszowski, Small Ramsey numbers, Electron. J. Combin. Dynamic Surveys DS1, Revision 14 (2014). 